\title{
El ordenamiento territorial para la gestión del riesgo de desastres en Colombia
}

\section{Land Management for Risk Management of Disasters in Colombia \\ O ordenamento territorial para a gestão do risco de desastres na Colômbia}

\author{
Daniel Calderón Ramírez* \\ Klaus Frey ${ }^{* *}$
}

Recibido: 18 de abril de 2016

Aprobado: 16 de mayo de 2016

Doi: http://dx.doi.org/10.12804/revistas.urosario.edu.co/territorios/a.4795

Para citar este artículo:

Calderón Ramírez, D. \& Frey, K. (2017). El ordenamiento territorial para la gestión del riesgo de desastres en Colombia. Territorios, (36), 239-264. Doi: http://dx.doi.org/10.12804/revistas.urosario.edu.co/ territorios/a.4795

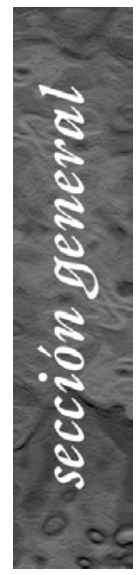

* Ecólogo, Pontificia Universidad Javeriana, Bogotá, Colombia. Magister en Desarrollo rural por la misma universidad. Estudiante de doctorado Universidad Federal del $A B C$, São Paulo, Brasil. Programa de Planificación y gestión del territorio. Correo electrónico: calderón.daniel@ufabc.edu. br. ORCID: http://orcid. org/0000-0003-1127-2602

** Graduado en Ciencias de la Administración, Universidad de Konstanz, Alemania. Doctor en Ciencias Sociales, Universidad de Konstanz, Alemania. Profesor titular Universidad Federal del ABC, São Paulo, Brasil. Programa de postrado en Planificación y gestión del territorio - Coordinador programa de posgrado politicas públicas. Correo electrónico: klaus.frey@ufabc.edu. br. ORCID: http://orcid. org/0000-0002-7564-1764 
Palabras clave

Ley Orgánica de

Ordenamiento

Territorial, Sistema

Nacional de Gestión del Riesgo de Desastres, Gobernanza del riesgo, Área Metropolitana del Valle de Aburrá.

Keywords

Land Management

Law, National

System for Disaster

Risk Management,

Risk Management, Metropolitan Area of the Aburrá Valley.

Palavras-chave

Lei Orgânica de Ordenamento Territorial, Sistema Nacional de Gestão do Risco de Desastres, Governança do risco, Área Metropolitana do Valle de Aburrá.

territarias 36 240

\section{RESUMEN}

En Colombia la Ley Orgánica de Ordenamiento Territorial (LOOT) es una de las herramientas que permitió la restructuración del Sistema Nacional de Gestión del Riesgo, generando que los entes territoriales pudieran organizarse en una gestión conjunta al nivel central, bajo los principios de descentralización, coordinación, complementariedad y concurrencia; esto con el objetivo de fomentar acciones estratégicas coordinadas hacia el conocimiento del riesgo, reducción del riesgo y manejo de la emergencia. De esta manera, la LOOT ha permitido mejorar las competencias de los entes territoriales para la gestión del riesgo de desastres, por medio del traspaso de mayor autonomía del nivel nacional hacia los departamentos y municipios, así como el fortalecimiento de asociaciones municipales como el caso del Área Metropolitana del Valle de Aburrá y su plan intermunicipal de gestión del riesgo — Red Riesgos-.

\section{ABSTRACT}

In Colombia, land management is one of the major tools that contributed to the restructuring of the National Risk Management System, enabling local authorities to organize a common management approach at the central level, based on the principles of decentralization, coordination, complementarity and competition. The main objective is to promote coordinated strategic actions in order to enhance risk awareness, to reduce concrete risks and to prepare for effective emergency management. Land management has contributed to improve the capacity of local authorities to manage disaster risks through the transfer of greater autonomy from the national level to the departments and municipalities, and finally by strengthening municipal associations, as in the case of the Metropolitan area of the Aburrá Valley, and its inter-risk management plan — Red Riesgos-.

\section{RESUMO}

Na Colômbia a Lei Orgânica de Ordenamento Territorial (LOOT), é uma das ferramentas que permitiu a restruturação do Sistema Nacional de Gestão do Risco, gerando que os entes territoriais pudessem organizar-se em uma gestão conjunta ao nível central, sob os princípios de descentralização, coordenação, complementariedade e concorrência; isto com o objetivo de fomentar ações estratégicas coordenadas ao conhecimento do risco, redução do risco e gestão da emergência. Desta maneira, a LOOT tem permitido melhorar as competências dos entes territoriais para a gestão do risco de desastres, por meio do traspasso de maior autonomia do nível nacional aos departamentos e municípios, assim como o fortalecimento de associações municipais como o caso da área Metropolitana do Valle de Aburrá e o seu plano intermunicipal de gestão do risco -Rede Riscos-. 


\section{Introducción}

En Colombia, la institucionalidad de la gestión del riesgo está conformada por un régimen normativo que coordina las relaciones entre el conjunto de entidades públicas, privadas y de la sociedad civil. La función del Estado, como ente regulador de riesgos, parte de la misión de asegurar la construcción de nación (Revéiz, 2013), esto por medio de la elaboración de instrumentos que permitan delegar funciones bajo un esquema de organización en los distintos ámbitos sectoriales y territoriales.

En el ámbito territorial, la institucionalidad para la gestión del riesgo se sustenta en los procesos, las estrategias y los proyectos específicos de corto, mediano y largo plazo realizados por los entes territoriales - departamentos, municipios y regiones del país - en los procesos de conocimiento del riesgo, reducción del riesgo y manejo del desastre. Esto se desarrolla bajo una estructura organizativa, unos instrumentos de planificación, unos sistemas de información y unos mecanismos de financiación.

Este tipo de organización para la gestión del riesgo muestra claramente los principios de la descentralización que según Ferrando (1992, citado en Trujillo, 2007), se describe como una pluralidad de órganos de decisión y de centros particulares de interés; una dirección de estas instituciones por autoridades independientes o semi-independientes; y una sujeción de tales autoridades a un control cualificado. De esta manera, la descentralización es uno de los principios para la gestión del riesgo. A pesar que los riesgos se presentan en lugares puntuales, su afectación y sus orígenes nunca son locales. Por esta razón, su gestión se amplía al nivel regional, nacional e internacional por medio de la creación de sistemas o plataformas que coordinan a las entidades y a los actores, generando una institucionalidad basada en los principios de la gobernanza de múltiples niveles. Esta coordinación multiescalar es posible gracias a los principios de una política de Ordenamiento Territorial.

En Colombia, la Ley Orgánica de Ordenamiento Territorial (LOOT) 1454 del 28 de junio de 2011 tiene como objetivo dictar las normas para la organización político-administrativa del territorio, por medio de la definición de un marco institucional que especifique las competencias del Estado y los entes territoriales, junto a los instrumentos de planificación que permitan el desarrollo territorial.

La finalidad del ordenamiento territorial es promover el aumento de la descentralización, la planeación, la gestión y la administración de los entes territoriales, con la correspondiente asignación de recursos, con la intención de propiciar las condiciones para concertar políticas públicas entre el Estado, el departamento y los municipios, donde se reconozcan las características particulares de cada territorio (Congreso de Colombia, 2011, p. 2).

De esta manera, la LOOT ha sido la base del ordenamiento territorial en $\mathrm{Co}^{-}$ territarias 36 241 
lombia, siendo uno de los instrumentos indispensables para la formulación de otras políticas públicas enfocadas al desarrollo territorial. El caso de la Ley 1523 del 24 de abril de 2012 , en la cual se adopta la política nacional de gestión del riesgo de desastres y se establece el Sistema Nacional de Gestión del Riesgo (SNGRD), bajo los enfoques de coordinación territorial, concurrencia y subsidiaridad, pone en evidencia la importancia del ordenamiento territorial para la gestión del riesgo de desastres. Según la ley 1523 (Art 1): “la gestión del riesgo de desastres es una política de desarrollo indispensable que asegura la seguridad territorial y mejora la calidad de vida de las poblaciones y las comunidades en riesgo" (p.l). Por tanto, la gestión del riesgo está ligada a la planificación del desarrollo seguro y a la gestión ambiental territorial sostenible.

Complementando a la LOOT se encuentra la ley 388 de 1997, que brinda directrices hacia el desarrollo y ordenamiento territorial municipal y distrital, incluyendo la gestión del riesgo de desastres como uno de los objetivos fundamentales para el ordenamiento del territorio donde la creación, protección y defensa del espacio público debe contener una visión preventiva hacia todo tipo de amenazas que afecten la integridad social y comprometan la sostenibilidad de la biodiversidad ecológica. Al mismo tiempo, esta ley establece, como requisito del ordenamiento territorial, la conservación y sostenibilidad de ecosistemas estratégicos con la finalidad de reducir los impactos ambientales negativos que conllevan al aumento de la vulnerabilidad territorial. De este modo, la ley intenta brindar a los entes territoriales directrices de sostenibilidad para la acción urbanística, que permitan continuar con los procesos de desarrollo sin aumentar el riesgo de desastres.

Uno de los principios que destaca la LOOT, fundamental para la formulación de planes de gestión del riesgo, son los esquemas asociativos territoriales, donde el Estado promueve procesos integradores de las entidades territoriales, nacionales e internacionales, para la libre y voluntaria conformación de alianzas estratégicas que impulsen el desarrollo territorial. Un ejemplo de asociación territorial para la planeación y gestión es el Área Metropolitana del Valle de Aburrá (AMVA), en el departamento de Antioquia, donde los gobiernos locales de diez municipios se articulan, evidenciando una mayor eficiencia y eficacia de la gestión del riesgo.

El presente artículo pretende describir los principios de la Ley Orgánica de Ordenamiento Territorial que han sustentado la función de coordinación que el Sistema Nacional de Gestión del Riesgo intenta realizar, junto a los diferentes entes territoriales, bajo una perspectiva de la gobernanza del riesgo. Así mismo, se resaltan las competencias de los entes territoriales y los instrumentos de planificación territorial, resaltando la asociación territorial como una estrategia de gestión del riesgo. En la primera parte se exponen los principios del ordenamiento territorial en Colombia. En la segunda parte se hace una breve 
alusión a los nuevos retos y paradigmas de la gestión del riesgo con énfasis en la gobernanza del riesgo. En la tercera parte se detalla la institucionalidad del Sistema Nacional de Gestión del Riesgo (SNGR). En la cuarta y última parte, se presenta el caso del Área Metropolita del Valle de Aburrá donde la asociación territorial ha sido una de las estrategias de gestión del riesgo de desastres.

\section{Los principios del Ordenamiento Territorial en Colombia}

Según Becker, Chica \& Cárdena (2003), el ordenamiento territorial hace parte del diseño de una estrategia político administrativa por parte del Estado, con el objetivo de lograr una articulación entre los intereses de la población, las instituciones y el territorio. "En otras palabras, se insiste en considerar el ordenamiento territorial como una condición para garantizar que la descentralización cumpla con los objetivos de alcanzar bienestar social, legitimar el sistema institucional y promover la productividad económica" (Becker et al., 2003 , p. 9). Desde este punto de vista, se considera al ordenamiento del territorio como una estrategia que permite mayor autonomía para el desarrollo económico y social de los entes territoriales, recalcando el papel que tiene el nivel local hacia la consecución de este objetivo. Esto permite legitimar las acciones de los territorios encaminadas en aprovechar y dinamizar las variables sociales, económicas, ecológicas y políticas propias de su entorno, permitiendo desarrollar ventajas comparativas y competitivas.

En Colombia, desde la Constitución de 1991 se considera al territorio como una dimensión fundamental para el desarrollo económico y social. Desde entonces, la Constitución brindó mayor legitimidad democrática a los entes territoriales, permitiendo ampliar las relaciones políticas entre la sociedad y el territorio que habita. De esta manera, el reconocimiento del municipio como la entidad fundamental de la división político-administrativa del Estado en el artículo 311 de Constitución puede llegar a cambiar el sentido del concepto de construcción de nación. La Ley 388 de 1997 en el artículo $5^{\circ}$ define el ordenamiento territorial municipal como:

un conjunto de acciones político-administrativas y de planificación física concertadas, emprendidas por los municipios o distritos y áreas metropolitanas, en ejercicio de la función pública que les compete, dentro de los límites fijados por la Constitución y las leyes, en orden a disponer de instrumentos eficientes para orientar el desarrollo del territorio bajo su jurisdicción y regular la utilización, transformación y ocupación del espacio, de acuerdo con las estrategias de desarrollo socioeconómico y en armonía con el medio ambiente y las tradiciones históricas y culturales (Congreso de Colombia, 1997, p. 2).

Entendido el ordenamiento territorial como una función pública para la acción territarias 36 243 
administrativa y la planificación, la gestión del riesgo de desastres naturales se convierte en unas de las líneas de acción fundamentales para continuar con el desarrollo territorial, mediante la utilización, transformación y ocupación del espacio. Por esta razón, la ley 388 determina que uno de los objetivos del ordenamiento territorial a nivel municipal es la gestión del riesgo de desastres y por lo tanto es necesario:

[...] el establecimiento de los mecanismo que permitan al municipio, en ejercicio de su autonomía, promover el ordenamiento de su territorio, el uso equitativo y racional del suelo, la prevención y defensa del patrimonio ecológico y cultural localizado en su ámbito territorial y la prevención de desastres en asentamiento de alto riesgo, así como la ejecución de acciones urbanísticas eficientes (Congreso de Colombia, 1997, p. 1).

Por lo tanto, la ley considera, entre otros aspectos, "mejorar la seguridad de los asentamientos humanos ante el riesgo de desastres naturales" (Congreso de Colombia, 1997, p. 2). Para este fin, la ley establece las competencias de la nación, los departamentos, las áreas metropolitanas y los municipios otorgando responsabilidad, a cada uno, sobre la planificación urbanoterritorial, el uso del suelo y la elaboración de planes y proyectos que contemplen la protección del medio ambiente y la seguridad de la población.

Según la Ley, dentro de las responsabilidades, de cada uno de los entes territoriales, en relación con el ordenamiento territorial y la gestión del riesgo de desastres naturales se desatanca: a) a la nación le compete el ordenamiento territorial de interés nacional como las áreas protegidas y los parques nacionales; b) a los departamentos les corresponde la elaboración de directrices y orientaciones para el ordenamiento territorial en función de los objetivos de desarrollo, potencialidades y limitantes biofísicos, económicos y culturales; y definir las políticas de asentamientos poblacionales en centros urbanos; c) al nivel metropolitano le corresponde elaborar el plan integral de desarrollo metropolitano así como definir las normas y criterios por las cuales los municipios se regularán; la delimitación de áreas protegidas estratégicas en la generación de bienes y servicios ambientales; d) los municipios deberán formular y adoptar los planes de ordenamiento territorial, reglamentar los usos del suelo a nivel urbano y rural, y coordinar los planes sectoriales como la gestión del riesgo de desastres con los planes a nivel departamental, metropolitano y nacional.

Según el artículo $8^{\circ}$ de la Ley 388 :

La función pública del ordenamiento del territorio a nivel municipal y distrital se ejerce mediante la acción urbanística referida a las decisiones administrativas y a las actuaciones urbanísticas que le son propias, relacionadas con el ordenamiento del territorio y la intervención en los usos del suelo. (Congreso de Colombia, 1997, p. 5)

Dentro de la acción urbanística relacionada con la gestión del riesgo de 
desastres, el conocimiento del riesgo con la determinación de los diferentes tipos de amenazas y vulnerabilidades, se convierten en una de las estrategias para la reducción del riesgo. Así mismo, se contempla la conservación y sostenibilidad de ecosistemas estratégicos que sustentan el equilibrio entre las relaciones sociedad y naturaleza.

1) determinar las zonas no urbanizables que presenten riesgos para la localización de asentamiento humanos, por amenazas naturales, o que de otra forma presenten condiciones insalubres para la vivienda 2) localizar las áreas críticas de recuperación y control para la prevención de desastres, así como las áreas con fines de conservación y recuperación paisajística. 3) Identificar y caracterizar los ecosistemas de importancia ambiental del municipio de acuerdo con la autoridad ambiental de la respectiva jurisdicción, para su protección y manejo adecuados (Congreso de Colombia, 1997, p. 6).

Por otra parte, la función del ordenamiento territorial, como un instrumento de planificación y de gestión de las entidades territoriales, está regulada por la Ley Orgánica de Ordenamiento Territorial (LOOT) 1454 de 2011. Los principios de la LOOT, están enfocados principalmente hacia: a) la promoción de una mayor delegación de funciones y competencias del nivel nacional hacia el orden territorial (Art, 21, 26, 27, $28,29)$; b) la eliminación de duplicidades de competencias entre la administración central y los entes territoriales (Art, 26, $27,28,29)$; c) el fortalecimiento de las
Regiones de Planeación y Gestión y las Regiones Administrativas y de Planificación (Art 10, 16, 19); d) el fortalecimiento del departamento como nivel intermedio (Art 22, 29); e) el fortalecimiento del municipio como entidad fundamental de la división político administrativa del Estado (Art 3, $29)$; f) la acción conjunta y articulada de los diferentes niveles de gobierno a través de alianzas, asociaciones y convenios de delegación (Art 29, 30); g) el diseño de modalidades regionales de administración para el desarrollo de proyectos especiales (Art 10, 12, 13, 14, 15, 16, 19); h) el incremento de la productividad y modernización de la administración municipal (Art 3, 29).

Según la Constitución Política de Colombia (1991), son entidades territoriales los departamentos (32), los distritos (6), los municipios (1123 en total) y los territorios indígenas. Así mismo, podrán llegar a constituirse entidades territoriales las regiones y provincias. El artículo 287 de la Constitución, dicta que las entidades territoriales gozan de autonomía para la gestión de sus intereses dentro de los límites de la Constitución y la Ley, respaldados por el principio de descentralización. "El proceso de descentralización colombiano se inició siguiendo la tendencia observada en América Latina, con la firme intención de mejorar el desempeño de los gobiernos subnacionales para contribuir al desarrollo económico y al mejoramiento de la calidad de vida" (Bonet, Pérez \& Ayala, 2014, p. 3 ). territarias 36

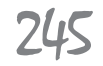




\subsection{Descentralización}

Colombia, bajo la Constitución de 1991, es una república unitaria donde se proclama el principio de descentralización, reconociendo la gran diversidad social y biofísica del país; con lo cual, se otorga autonomía a los entes territoriales para liderar su propio desarrollo mediante el aprovechamiento de sus ventajas competitivas y comparativas. Para que cada territorio pueda llevar a cabo este propósito, la Constitución define la descentralización como principio de la acción de todas las ramas del poder público y, en especial, de la Administración Pública. De esta manera, se otorga un traspaso de funciones de un nivel de gobierno a otro, otorgando competencias administrativas, fiscales y políticas (Manrique, 2010; Revéiz, 2013).

Las reformas de descentralización pueden atribuirse a una crisis de gobernabilidad y legitimidad del Estado colombiano, como lo afirma Cabrera \& Naranjo (2003) quienes, a través del análisis de estudios realizados por el Departamento Nacional de Planeación (DNP), afirman que Colombia se caracterizó, durante la administración centralista, por las restricciones a la democracia, el deterioro en la provisión de bienes y servicios públicos locales, especialmente en los municipios más pequeños y atrasados. Esto conllevó a la búsqueda de mecanismos más eficientes en la asignación de recursos, basados en la teoría económica y el federalismo fiscal.

A pesar de este eclecticismo político,

Colombia se ha constituido como un Esta- do Unitario Descentralizado, concepto que describe Trujillo (2007), como una forma institucional del centralismo político, en la cual todos los atributos del poder emanan de una sola jerarquía constitucional que se proyecta sobre la organización general del Estado pero, con la coexistencia de centros diversos, institucionalmente organizados para el impulso y el ejercicio de la administración pública.

De las relaciones entre autoridades centrales y descentralizadas se despenden los conceptos de descentralización política, económica administrativa y fiscal, cuyos ámbitos pueden manejarse de forma complementaria o en forma independiente como lo explica Trujillo (2007). En términos de una descentralización integral, el traslado de competencias abarca todos los campos referidos. Sin embargo, puede haber descentralización administrativa con centralización fiscal

Según Falleti (2010), la autonomía de los entes territoriales en las competencias administrativas, fiscales y políticas no se presentan simultáneamente. En el caso colombiano, primero se produjo una descentralización política, seguida de una fiscal y administrativa. Siguiendo a Falleti, el orden en el cual se produce cada una de las autonomías, repercute finalmente en el grado de autonomía administrativa. Según él, si bien Colombia obtuvo en primera medida una autonomía política, a nivel administrativo y fiscal muchos de los entes territoriales actualmente tienen una dependencia del nivel central. 


\subsection{Descentralización fiscal}

Las entidades territoriales, para llevar a cabo el proceso de descentralización, participan de las rentas nacionales por medio del Sistema General de Participaciones (SGP), por el cual, la Nación transfiere recursos para la financiación de las competencia estatales, asignadas en la Ley 715 de 2001, referentes principalmente a educación, salud, saneamiento básico y disposiciones generales como la gestión del riesgo de desastres naturales.

El SGP es asignado a los municipios dependiendo de la categoría municipal a la que pertenezcan, la cual está relacionada principalmente con el número de habitantes. Los municipios con menos de 50 mil habitantes dependen casi completamente del SGP. En estos municipios las transferencias nacionales representan el $80 \%$ o más de los ingresos corrientes. En el caso de los municipios mayores de 500 mil habitantes, las transferencias representan el $40 \%$ de sus ingresos, proporción que aumenta a $55 \%$ en la categoría de 100 a 500 mil habitantes, y a $68 \%$ en la categoría de 50 a 100 mil (Manrique, 2010).

Aparte de la SGP, la autonomía de los entes territoriales depende de las fuentes de financiamiento propios a través de la recaudación de impuestos. En tal sentido, las leyes tributarias han determinado que los impuestos más productivos - la renta y el Impuesto de Valor Agregado (IVA) estén en manos del nivel central mientras que las entidades territoriales gozan de fuentes tributarias menos dinámicas, como lo explica Cabrera et al. (2003). En el caso de los departamentos estos pueden recaudar impuestos sobre los licores, tabaco, la suerte y el azar (loterías). Los municipios, por su parte, gozan de fuentes tributarias un poco más dinámicas como los impuestos a la propiedad inmueble, prediales, impuestos de la industria y comercio y a la gasolina.

\subsection{Competencias}

En la LOOT se entiende como competencia la facultad que tiene la Nación, las entidades territoriales y las figuras de integración territorial para atender de manera general responsabilidades estatales. Los departamentos y municipios tienen autonomía para determinar su estructura interna y organización administrativa central y descentralizada; así como el establecimiento y distribución de sus funciones y recursos para el adecuado cumplimiento de sus deberes constitucionales.

Según el artículo 27 de la LOOT, para la asignación de competencias se tienen en cuenta principalmente los principios de: a) coordinación: donde la Nación y las entidades territoriales deben ejercer sus competencias de manera articulada y armónica, por esto, las entidades territoriales se articularan con las autoridades nacionales; b) subsidiaridad: donde las autoridades de planeación del nivel más amplio deberán apoyar transitoriamente a aquellas que carezcan de capacidad técnica para la preparación oportuna del plan de desarrollo; c) complementariedad: en el ejercicio de las territarias 36 247 
competencias en materia de planeación, las autoridades actúan colaborando con otras autoridades, dentro de su órbita funcional, con el fin de que el desarrollo de aquéllas tenga plena efectividad; d) concurrencia: se presenta cuando dos o más autoridades de planeación deben desarrollar actividades en conjunto hacia un propósito $\mathrm{co}^{-}$ mún, donde su actuación tiene facultades en distintos niveles con el propósito de ser oportunos eficientes y eficaces. Según Trujillo (2007), las competencias entre el Estado central y los entes territoriales se rigen por el principio de exclusividad, el cual determina que la función asignada a un ente no pueda ser ejercida por otro, sin prejuicio de la vigencia de los principio de coordinación y subsidiaridad.

\subsection{Regionalización}

Jurídicamente la región es una entidad territorial cuya existencia es producto de una construcción política que, como lo explica Trujillo (2007), es más que el espacio físico: se refiere a las instituciones que gobiernan a una población con intereses comunes, asentada en un territorio delimitado por fronteras que en un momento dado pueden no depender de la geografía sino de las decisiones humanas. Según Ferrando (1992, citado en Trujillo, 2007) la región, desde el punto de vista sociológico, establece tres elementos centrales: un elemento espacial, dado por las características biofísicas embarcadas dentro de un contexto de apropiación socio-política; una conciencia regional: refiriéndose a la existencia de una identidad socio-cultural; una tendencia a una organización social: como una vocación colectiva para adoptar formas comunes de institucionalidad.

Según Uribe (1998), la regionalización es analizada como un nivel intermedio necesario para la conexión entre el nivel local y nacional. Este criterio se apoya en que la planificación nacional y local, actuando de forma independiente, serían incapaces de crear condiciones para manejar en forma adecuada y eficiente las políticas públicas de desarrollo. De esta manera, 1) las políticas nacionales nunca podrán considerar la diversidad de situaciones regionales; 2) la planificación local opera en un sistema muy pequeño; 3 ) ni el ámbito nacional ni el local están en condiciones de percibir la especificidad de las estructuras económicas, sociales y políticas de carácter regional y sus coyunturas de desarrollo; 4) sin un sistema regional sería muy difícil obtener un grado significativo de coordinación entre los distintos planes de ámbito local.

Según la LOOT, la autonomía que brinda el Estado a los entes territoriales, les da la facultad de establecer regiones de planeación y gestión, generando regiones donde se establecen relaciones geográficas, económicas y funcionales a partir de la noción de complementariedad. Así mismo, el Gobierno Nacional, a través de incentivos fiscales, promueve la conformación de esquemas asociativos como: regiones administrativas y de planificación, regiones de planeación y gestión, provincias administrativas y de planificación, y áreas metropolitanas. La regionalización tiene 
como objetivo que las entidades territoriales económicamente desarrolladas se asocien con las más débiles, con el fin de hacer efectivos los principios de subsidiaridad, equidad territorial, equidad social, sostenibilidad ambiental y equilibrio territorial. Igualmente, el Estado promueve procesos asociativos entre entidades territoriales nacionales y las de países vecinos y fronterizos tendientes a la conformación de alianzas estratégicas que promuevan el desarrollo territorial.

De esta manera, los principios de la LOOT permiten que el Estado colombiano pueda abordar la gestión del riesgo de desastres naturales desde una visión territorial, permitiendo una coordinación multinivel que puede conllevar a reducir la vulnerabilidad social, por medio de la organización y actuación de la sociedad. Esto permite que los municipios aumente la participación de la sociedad civil en sus sistemas de gestión del riesgo generando una gobernanza del riesgo.

\section{Los nuevos enfoques de la gestión del riesgo}

El interés mundial en la gestión del riesgo de desastres, presente en los últimos 30 años, ha ampliado la discusión sobre la gestión del desastre enfocada principalmente al estudio de fenómenos biofísicos, hacia una visión sistémica más compleja donde se reconocen relaciones entre diversas variables sociales y biofísicas, dentro de múltiples escalas de tiempo y espacio. Este nuevo abordaje, producto de una construcción social con diferentes visiones del fenómeno, permite analizar las características del riesgo, anteriormente ignoradas, relacionadas con el carácter de complejidad e incertidumbre, generando una percepción más amplia del riesgo con nuevas estrategias para gestionarlo.

En primer lugar, a nivel mundial, pero específicamente en América Latina y el sudoeste asiático, el riesgo empieza a ser analizado como un producto de procesos sociales particulares, donde influye de manera directa o indirecta el modelo de desarrollo de cada sociedad. De esta manera, la identificación de la vulnerabilidad social empieza a ser una pieza esencial en el análisis del riesgo donde la pobreza, la desigualdad social, el analfabetismo, la corrupción y la estructura de gobierno son variables sociales que necesitan ser tenidas en cuenta con el objetivo de generar resiliencia y adaptación territorial, conceptos que empiezan a ser asociados a fenómenos como el cambio climático.

En un segundo aspecto, bajo este enfoque sistémico, el riesgo deja de ser considerado como un fenómeno estrictamente local. El liberalismo económico y la consecuente globalización crearon una económica mundial con una amplia distribución y, por lo tanto, un alto grado de exposición a amenazas biofísicas. El sudoeste asiático, donde existen grandes inversiones de capital financiero mundial, es una de las regiones del mundo con mayor índice de riesgo de desastres naturales debido a la alta probabilidad de amenazas biofísicas y altas vulnerabilidades como pobreza, territarias 36 249 
ausencia de infraestructura y carencia de gobiernos organizados para enfrentar el desastre. De esta manera, la afectación no solo se presenta en los países que sufren el desastre, el riesgo se convierte en global y los efectos del desastre, en cualquier lugar del mundo, repercuten en el sistema económico mundial. Las nuevas concepciones del riesgo tienen en cuenta aspectos como la globalización, las dinámicas del sistema económico mundial, la desigualdad social y los tratados socioeconómicos.

En un tercer lugar, se encuentran las nuevas perspectivas de los sistemas de gestión donde hay una redistribución de responsabilidades, estableciendo acuerdos de cooperación entre múltiples actores. La antigua visión técnica y burocrática de la gestión del desastre, centralizada y dirigida por especialistas que intentaban actuar bajo la incertidumbre, la complejidad y la ambigüedad del riesgo, pretende ser reemplazada por sistemas de gestión donde la sociedad civil tiene una mayor participación y protagonismo en procesos de prevención y reducción del riesgo. Mediante la organización comunitaria, la sociedad civil pasó a tener un papel preponderante en los sistemas de alerta, la detección y reconocimiento de amenazas, la actuación durante la emergencia, la educación ambiental y la participación en sistemas de seguridad alimentaria, resultado de diferentes aproximaciones dentro del paradigma de la gobernanza del riesgo. tersitarias 36

\subsection{La gobernanza del riesgo}

La gobernanza del riesgo ha cambiado la visión tradicional de gestión del riesgo, donde el Estado actuaba de una forma jerárquica y centralizada para organizar las agencias gubernamentales, hacia un sistema policéntrico en el cual la política se distribuye hacia diferentes niveles de gobierno y escalas territoriales. Esto implica un incremento de la participación de diversos agentes donde es posible tener en cuenta su percepción, evaluación, diversidad de conocimiento e intereses políticos en el orden de producir análisis de la vulnerabilidad y toma de decisiones en la gestión del riesgo. Esta diversidad institucional puede ofrecer considerables ventajas en la complejidad, incertidumbre científica y ambigüedad socio política del riesgo, donde es posible tener diversas miradas desde diferentes niveles con el fin de tener una mayor resiliencia y una menor vulnerabilidad.

Según Klinke \& Renn (2013), este enfoque permite enfrentar la complejidad de las características de la gestión del riesgo como: a) la ausencia de información y conocimiento del riesgo, donde se resalta la dificultad de identificar y cuantificar la vulnerabilidad de la sociedad y las potenciales amenazas que podrían llegar a afectarla según el nivel de exposición; b) la incertidumbre científica y reducción del riesgo, que referencia la probabilidad en que ocurra la amenaza sin lograr anticiparse al desastre; y c) ambigüedad socio-política y manejo del desastre como 
resultado de la ausencia de información e incertidumbre, produciendo una percepción social del riesgo desligada a la realidad y que puede estar sujeta a una mercantilización del riesgo propiciada por intereses económicos y/o políticos. Esto conduce a una ambigüedad normativa donde la percepción del riesgo genera políticas que pueden estar lejanas a la realidad.

Según Klinke et al. (2013) la gobernanza del riesgo debe enfocarse a la planificación territorial a través de cinco fases: 1) Pre-evaluación del riesgo, donde se busca determinar la percepción del riesgo de la sociedad; indagar sobre los tipos de vulnerabilidad social, ambiental, política y económica; así como establecer las posibles amenazas y el grado de afectación que pudiera existir. 2) Evaluación interdisciplinar del riesgo, busca caracterizar a la sociedad tratando de establecer los diferentes tipos de actores y organizaciones sociales relacionadas con la gestión del riesgo, identificando posibles aliados, conflictos, intereses comunes, interdependencias y potenciales interacciones, tratando de establecer conexiones entre la comunidad científica, el sector de la tecnología, el sector público y el sector privado. 3 ) Evaluación del riesgo, enfocado a determinar la probabilidad de las amenazas y el nivel de impacto que tendrían sobre el estado de vulnerabilidad y el grado de exposición de la sociedad. 4) Gestión del Riesgo donde se ponen en práctica las anteriores fases descritas incluyendo un plan de acción que permita enfocar la gestión del riesgo bajo el conocimiento del riesgo, reducción del riesgo y manejo de la emergencia. 5) Comunicación del riesgo, pretende encontrar espacios y canales de comunicación efectivos con la finalidad de producir participación y consenso de la sociedad civil en la toma de decisiones.

La gobernanza del riesgo es un concepto que empieza ser ampliamente discutido e implementado, sobretodo en ciudades, al mismo tiempo que surgen nuevas investigaciones que evalúan la eficacia de este tipo de arreglo institucional. Hasta ahora, las discusiones académicas realizadas giran en torno a analizar la relación entre la estructura político administrativa del Estado y la gobernanza del riesgo, tratando de identificar cuál es la organización administrativa ideal del Estado para implementar este tipo de arreglo institucional.

\section{El Sistema Nacional de Gestión del Riesgo de Desastres (SNGRD)}

La nación colombiana, debido a su ubicación geográfica, tiene una gran diversidad geológica, geomorfológica, hidrológica y climática, convirtiéndose en uno de los países con mayor grado de exposición a amenazas naturales (UNGRD, 2013). Ante el riesgo de desastres naturales, Colombia ha formulado una reglamentación que ha ido evolucionando con el aprendizaje y la coordinación internacional. La primera reglamentación data del año 1988 en el que se creó el Sistema Nacional para la Prevención y Atención de Desastres. En el año 1989, por medio del Decreto Ley 919, se crearon los Comités Regionales para la Prevención y Atención de Desastres territarias 36

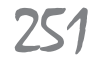


(CREPAD) y los Comités Locales para la Prevención y Atención de Desastres (CLOPAD).

Por otro lado, Colombia suscribió acuerdos internacionales que fortalecieron los principios políticos de la gestión del riesgo como lo fue la Declaración de Río de Janeiro de 1992, con énfasis en el reconocimiento de acciones frente al cambio climático; el Marco de Acción de Hyogo 2005-2015, donde se concluye que la prevención y mitigación debe ser realizadas por medio de la preparación ante los riesgos, la reducción de la vulnerabilidad y la necesidad del fortalecimiento institucional; y el Marco de Acción de Sendai 20152030, que busca reducir la vulnerabilidad social, sobre todo la pobreza, con el objetivo de reducir el número de víctimas en el mundo por desastres naturales. Sin embargo, en Colombia hasta el año 2012 no existía una política nacional para la gestión del riesgo de desastres naturales; hasta entonces, el Estado formulaba instrumentos de planeación que se relacionaban directa o indirectamente con la gestión del riesgo.

Entre los años 2010 y 2012 Colombia resultó afectada por un episodio muy fuerte del fenómeno de La Niña. De las 1041 municipalidades de Colombia, 93 resultaron perjudicadas, especialmente por las diversas inundaciones (OSSO, 2012, citado en UNISDR, 2013). Se calculó que las pérdidas económicas directas ascendieron a unos 6 mil millones de dólares americanos, lo cual representó casi el $6 \%$ de la formación bruta de capital del país (CEPAL, 2012 citado en UNISDR, 2013)
Frente al agudo fenómeno climático se replanteó la Política Nacional de Gestión del Riesgo bajo la ley 1523 del 24 de abril del 2012 y se reestructuró el Sistema Nacional de Gestión del Riesgo de Desastres (SNGRD), poniendo en disposición una estructura de coordinación territorial a través de la Unidad Nacional de Gestión del Riesgo de Desastres (UNGRD) con el objetivo de que la gestión del riesgo sea un proceso social orientado a la formulación, ejecución, seguimiento y evaluación de políticas, estrategias, planes, programas, regulaciones, instrumentos, medidas y acciones permanentes para el conocimiento del riesgo, la reducción del riesgo y el manejo del desastre.

Las instancias públicas de orientación y coordinación del Sistema Nacional de Gestión del Riesgo de Desastres (SNGRD), están organizadas de forma descentralizada, jerárquica y burocrática, a la cabeza de la presidencia de la república, el cual está orientado por el Consejo Nacional para la Gestión del Riesgo, que está compuesto por el presidente de la república, una junta de la que hacen parte todos ministerios públicos, el director del Departamento de Planeación Nacional (DNP) y el director de la Unidad Nacional de Gestión del Riesgo. Este Consejo dirige y coordina la Unidad Nacional de Gestión del Riesgo y Desastres quienes son asesorados técnicamente por el Comité Nacional del Conocimiento del Riesgo, el Comité Nacional para la Reducción del Riego y el Comité Nacional de Manejo del Desastre. Estos comités, principalmente de carácter técni- 
co, asesoran a los comités departamentales y municipales de conocimiento del riesgo, reducción del riesgo y manejo del desastre. Los gobernadores departamentales hacen seguimiento y control a la implementación de la gestión del riesgo en los municipios, mediante los instrumentos de planificación de gestión del riesgo. En la tabla 1, se especifican las funciones de los entes territoriales en el Sistema Nacional de Gestión del Riesgo.

El Sistema de Gestión del Riesgo es un sistema de coordinación abierto, compuesto por entidades públicas, privadas con ánimo y sin ánimo de lucro y la sociedad civil. Para lograr contar con la participación de la sociedad civil, el Estado colombiano formuló la Ley 1505 del 5 de enero de 2012, por medio de la cual se crea el Subsistema Nacional de Voluntarios de Primera Respuesta, donde se otorgan estímulos a los voluntarios de la Defensa Civil, a los Cuerpos de Bomberos y a la Cruz Roja. Así mismo, propuso la Ley 1575 del 21 de agosto de 2012, ley general de bomberos. También elaboró el Plan Nacional de Formación y Capacitación del Sistema Nacional de Gestión de Riesgo de Desastres, que tiene el fin de mejorar los niveles de percepción del riesgo, generar corresponsabilidad ciudadana y aumentar los niveles de participación que logren movilizar a la sociedad en torno a la gestión del riesgo.

La Ley Nacional de Gestión del Riesgo es considerada como esencial dentro de la planificación del desarrollo y por esto debe ser tenida en cuenta, a nivel departamental y municipal, en los Planes de Ordena- miento Territorial (POT), los Planes de Ordenamiento de Cuencas Hidrográficas (PONCAS) y los Planes de Desarrollo. En estos instrumentos de planificación se debe realizar e integrar un análisis del riesgo en el diagnóstico biofísico, económico y socio-ambiental, considerando el riesgo de desastres, como un condicionante para el uso y la ocupación del territorio. Es así como la gestión del riesgo está asociada con la planificación del desarrollo seguro, por medio de la gestión ambiental territorial sostenible, el involucramiento de todos los niveles de gobierno y la efectiva participación de la población.

Adicionalmente, la Unidad Nacional de Gestión del Riesgo, mediante el uso de Sistemas de Información Geográfica (SIG), desarrolló un sistema nacional de información, el cual se actualiza mediante la integración de contenidos de todas las entidades nacionales y territoriales. Este sistema tiene el propósito de fomentar la generación y uso de información sobre el riesgo de desastres generando estrategias de reducción y respuesta a emergencias en el territorio nacional.

\subsection{El Fondo Nacional de Gestión del Riesgo}

El Sistema de Gestión del Riesgo cuenta con un Fondo Nacional de Gestión del Riesgo, a cargo del Director de la Unidad Nacional de Gestión del Riesgo, el cual es un fideicomiso estatal de creación legal constituido como patrimonio autónomo. Fue creado con fines de interés público y territarias 36

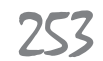


asistencia social con el propósito de atender las necesidades que se originen en situaciones de desastre o calamidad. Dentro de sus principales objetivos están los de negociar, obtener, recaudar, administrar, invertir, gestionar instrumentos de protección financiera y distribuir los recursos necesarios para la implementación de la política de Gestión del Riesgo en donde están incluidos los procesos de conocimiento del riesgo, reducción del riesgo y manejo del desastre.

Para este fin, el Fondo Nacional recibe, administra e invierte recursos que provienen del Estado, así como, aportes hechos por personas naturales o jurídicas e instituciones del orden público y privado. El Gobierno Nacional, a través del Ministerio de Hacienda y Crédito Público, garantiza que en todo momento el Fondo Nacional cuente con recursos suficientes que permitan asegurar el apoyo a las entidades nacionales y territoriales. Los recursos del Fondo están sujetos a la asignación en el Presupuesto General de la Nación y están contenidos en el Marco de Gastos de Mediano Plazo (MGMP). El Director de la Unidad Nacional de Gestión del Riesgo establece la distribución de estos recursos en las subcuentas de conocimiento del riesgo, reducción del riesgo y manejo del desastre, de acuerdo con las prioridades que se determinan en cada uno de los procesos de la gestión del riesgo.

Las administraciones departamentales, distritales y municipales, deben tener sus propios fondos de gestión del riesgo bajo el esquema del Fondo Nacional, como cuentas especiales con autonomía técnica y financiera, con el propósito de invertir, destinar y ejecutar sus recursos en la adopción de medidas de conocimiento y reducción del riesgo de desastre, preparación, respuesta, rehabilitación y reconstrucción. De esta forma, los entes territoriales pueden establecer mecanismos de financiación dirigidos a las entidades involucradas en los procesos y a la población afectada por la ocurrencia de desastres o calamidad.

\subsection{Las competencias departamentales en la gestión del riesgo}

La formulación del Plan Departamental de Gestión del Riesgo es responsabilidad de la autoridad territorial. Según la guía metodológica para la elaboración de planes departamentales de gestión del riesgo (UNGR, 2013), el plan departamental es la herramienta a través de la cual se priorizaran programas y se ejecutan acciones hacia el conocimiento del riesgo, la reducción del riesgo y el manejo del desastre. De esta manera, el departamento debe realizar una identificación de: a) el contexto territorial, teniendo en cuenta las características físicas, sociales, económicas y ambientales, así como las institucionales; b) el inventario de las herramientas, instrumentos, programas, marcos jurídicos y recursos humanos con los que cuanta el departamento para realizar el plan; c) los factores de riesgo, tipos de amenazas, vulnerabilidades y grados de exposición presentes en el territorio y análisis del riesgo; d) la caracterización 
Tabla 1. Sistema Nacional de Gestión del Riesgo de Desastres en Colombia

\begin{tabular}{|c|c|c|c|c|c|}
\hline & $\begin{array}{l}\text { Responsables } \\
\text { directos }\end{array}$ & Competencias a nivel nacional & $\begin{array}{c}\text { Competencias a nivel } \\
\text { departamental }\end{array}$ & $\begin{array}{c}\text { Competencias a nivel } \\
\text { municipal }\end{array}$ & Planificación \\
\hline 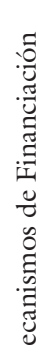 & $\begin{array}{l}\text { Conocimiento del } \\
\text { riesgo: a través del } \\
\text { Consejo nacional } \\
\text { y los comités } \\
\text { departamentales } \\
\text { y municipales de } \\
\text { conocimiento del } \\
\text { riesgo. }\end{array}$ & $\begin{array}{l}\text { 1 Formulación de políticas } \\
\text { que fortalezcan el proceso de } \\
\text { conocimiento del riesgo en el } \\
\text { país. } \\
2 \text {. Orientar y apoyar las } \\
\text { competencias de los entes } \\
\text { territoriales a nivel de } \\
\text { conocimiento del riesgo. }\end{array}$ & $\begin{array}{l}\text { 1. Identificación de } \\
\text { escenarios de riesgo en } \\
\text { sus diferentes factores, } \\
\text { entiéndase: amenazas, } \\
\text { vulnerabilidades, } \\
\text { exposición de personas } \\
\text { y bienes. }\end{array}$ & $\begin{array}{l}\text { 1. Generación de análisis } \\
\text { y evaluación del riesgo. } \\
\text { 2. Establecer acciones de } \\
\text { monitoreo y seguimiento } \\
\text { del riesgo y sus factores. } \\
\text { 3. Establecer acciones de } \\
\text { comunicación de la existencia, } \\
\text { alcance y dimensión del } \\
\text { riesgo al sistema nacional y la } \\
\text { sociedad en general. }\end{array}$ & \multirow{3}{*}{$\begin{array}{l}\text { Plan Nacional, } \\
\text { Departamental } \\
\text { y Municipal de } \\
\text { Gestión del Riesgo } \\
\text { de Desastres, Planes } \\
\text { de Desarrollo, Planes } \\
\text { de Ordenamiento } \\
\text { Territorial, Planes } \\
\text { de Ordenamiento } \\
\text { de Cuencas } \\
\text { Hidrográficas, } \\
\text { Instrumentos } \\
\text { de planificación: } \\
\text { Presupuestos } \\
\text { nacional, } \\
\text { departamentales y } \\
\text { municipales; Fondo } \\
\text { financiero para la } \\
\text { gestión del riesgo de } \\
\text { desastres Sub-cuentas } \\
\text { para el conocimiento } \\
\text { del riesgo, reducción } \\
\text { del riesgo, manejo } \\
\text { del desastre y } \\
\text { recuperación; } \\
\text { Banco de Proyectos. } \\
\text { Estrategia Municipal } \\
\text { de Respuesta a } \\
\text { Emergencias - Planes } \\
\text { escolares para la } \\
\text { gestión del riesgo. }\end{array}$} \\
\hline 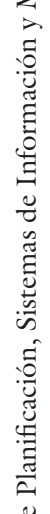 & $\begin{array}{l}\text { Reducción del } \\
\text { riesgo: a través del } \\
\text { Consejo nacional } \\
\text { y los comités } \\
\text { departamentales } \\
\text { y municipales de } \\
\text { reducción del riesgo. }\end{array}$ & $\begin{array}{l}\text { 1. Orientar la formulación de po- } \\
\text { líticas que fortalezcan el proceso } \\
\text { de reducción del riesgo en el país. } \\
\text { 2. Orientar y articular las políticas } \\
\text { y acciones de gestión ambiental, } \\
\text { ordenamiento territorial } \\
\text { planificación del desarrollo y } \\
\text { adaptación al cambio climático } \\
\text { que contribuyan a la reducción } \\
\text { del riesgo de desastres. } \\
\text { 3. Orientar la aplicación de } \\
\text { mecanismo de protección } \\
\text { financiera, entiéndase: seguros, } \\
\text { créditos, fondos de reserva, } \\
\text { bonos CAT, entre otros. }\end{array}$ & $\begin{array}{l}\text { 1. Generar acciones } \\
\text { de intervención } \\
\text { correctiva en las } \\
\text { condiciones existentes } \\
\text { de vulnerabilidad y } \\
\text { amenaza. } \\
\text { 2. Establecer la } \\
\text { intervención prospectiva } \\
\text { para evitar nuevas } \\
\text { condiciones de riesgo. }\end{array}$ & $\begin{array}{l}\text { 1. Establecer estrategias de } \\
\text { regulación técnica dirigidas a } \\
\text { la reducción del riesgo. }\end{array}$ & \\
\hline 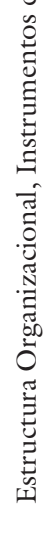 & $\begin{array}{l}\text { Manejo del } \\
\text { desastre: a través del } \\
\text { Consejo nacional } \\
\text { y los comités } \\
\text { departamentales } \\
\text { y municipales de } \\
\text { manejo del desastre. }\end{array}$ & $\begin{array}{l}\text { 1. Orientar la formulación de } \\
\text { políticas que fortalezcan el } \\
\text { proceso de manejo de desastre. } \\
\text { 2. Asesorar la formulación de la } \\
\text { estrategia nacional de respuesta a } \\
\text { emergencias. } \\
\text { 3. Asesorar la ejecución de } \\
\text { la respuesta a situaciones de } \\
\text { desastre con el propósito de } \\
\text { optimizar la atención a la } \\
\text { población, los bienes, ecosistemas } \\
\text { e infraestructura y la restitución } \\
\text { de los servicios esenciales. } \\
\text { 4. Orientar la preparación para } \\
\text { la recuperación, entiéndase: } \\
\text { rehabilitación y reconstrucción. }\end{array}$ & $\begin{array}{l}\text { 1. Establecer la } \\
\text { estrategia departamental } \\
\text { de respuesta a } \\
\text { emergencias. } \\
\text { 2. Formular la estrategia } \\
\text { departamental de } \\
\text { respuesta a situaciones } \\
\text { de desastre. } \\
\text { 3. Establecer la } \\
\text { estrategia para } \\
\text { la recuperación, } \\
\text { entiéndase: } \\
\text { rehabilitación } \\
\text { y reconstrucción. }\end{array}$ & $\begin{array}{l}\text { 1. Establecer la estrategia } \\
\text { municipal de respuesta a } \\
\text { emergencias. } \\
\text { 2. Formular la estrategia } \\
\text { municipal de la respuesta a } \\
\text { situaciones de desastre con } \\
\text { el propósito de optimizar } \\
\text { la atención a la población, } \\
\text { los bienes, ecosistemas e } \\
\text { infraestructura y la restitución } \\
\text { de los servicios esenciales. } \\
\text { 3. Establecer la estrategia } \\
\text { para la recuperación, } \\
\text { entiéndase: rehabilitación y } \\
\text { reconstrucción. }\end{array}$ & \\
\hline
\end{tabular}

Fuente: Elaboración propia, a partir de Banco Mundial, 2012 y Ley 1553 de 2012. 
de los escenarios de riesgo y priorización de las zonas de acción. Dado el caso, en que el departamento no tenga los recursos financieros o técnicos para realizar dicho diagnóstico, este podrá solicitarlos al nivel central o a otro ente territorial bajo los principios de subsidiaridad, concurrencia y complementariedad.

\subsection{Las competencias municipales en la gestión del riesgo}

Según la guía metodológica "Formulación del plan municipal de gestión del riesgo", intrumento de gestión del riesgo elaborado por la UNGRD (2012), el municipio debe generar el Plan de Gestión del Riesgo, un instrumento mediante el cual se formula, programa y hace seguimiento a la ejecución de las acciones que concretan los procesos de conocimiento del riesgo, reducción del riesgo y de manejo de desastres de forma articulada con los demás instrumentos de planeación municipal como: el plan de ordenamiento territorial, el plan de desarrollo, las agendas ambientales, los planes de acción de las diferentes entidades, instituciones y organizaciones que con su misión contribuyen al desarrollo social y económico del municipio.

El Plan de Gestión del Riesgo está formado por dos componentes: el primer componente de diagnóstico, que pretende realizar una caracterización general de los escenarios de riesgo donde se describen las condiciones de riesgo del municipio de manera general e identifica medidas de intervención alternativas siguiendo el esquema de procesos de la gestión del riesgo y un segundo componente programático, donde se define el impacto o cambio que se espera introducir para el desarrollo del municipio, los resultados que se deben obtener para lograr ese cambio y las acciones concretas que se deben ejecutar para lograr los resultados propuestos, definiendo alcances, responsables y costos, entre otros aspectos. Estos componentes son elaborados por el Consejo Municipal para la Gestión del Riesgo de Desastres (CMGRD) conformado por un grupo de personas de las entidades, instituciones y organizaciones públicas, privadas y comunitarias, quienes tienen el criterio y la autoridad para orientar el desarrollo municipal según las condiciones de riesgo presentes y futuras.

Adicionalmente el municipio debe elaborar la Estrategia Municipal para la Respuesta a Emergencias (EMRE) la cual es el marco de actuación para ejecutar los servicios de respuesta a emergencias de manera oportuna y efectiva. El objetivo de la EMRE es la efectividad en la prestación de los servicios de respuesta. La efectividad de la respuesta depende de la calidad de preparación, la cual consiste en acciones previas de coordinación, implementación de sistemas de alerta, capacitación, equipamiento, instalación de centros de reserva, albergues y entrenamiento. Estas actividades previas, implican financiamiento y deben ser objeto de planeación en virtud de los escenarios de riesgo y prioridades municipales. Así mismo, el municipio debe elaborar el Plan de Acción Específico para la Recuperación (PAER) que contiene 
las acciones para rehabilitar y reconstruir según sea el daño y sus opciones de recuperación en perspectiva del desarrollo sostenible.

\subsection{La planificación territorial para la gestión del riesgo}

Según la Ley 388 de 1997, el plan de ordenamiento territorial (POT) es el instrumento básico para el proceso de ordenamiento del territorio municipal. El artículo $9^{\circ}$ define el ordenamiento territorial como: "El conjunto de objetivos, directrices, políticas, estrategias, metas, programas, actuaciones y normas adoptadas para orientar y administrar el desarrollo físico del territorio y la utilización del suelo" (Congreso de Colombia, 1997, p. 6). $\mathrm{El}$ artículo $10^{\circ}$ establece que los planes de ordenamiento territorial a nivel municipal y distrital deben tener en cuenta "la conservación y protección del medio ambiente, los recursos naturales y la prevención de amenazas y riesgos naturales" (Congreso de Colombia, 1997, p. 7)

De esta manera, los POT tienen en cuenta, entre otros aspectos, las directrices y normas expedidos por el Sistema Nacional Ambiental, la ley 99 de 1993 y el Código de Recursos Naturales, así mismo las disposiciones de las Corporaciones Autónomas Regionales (CAR) concernientes a cada uno de los territorios. Con relación a la gestión del riesgo de desastres naturales el POT incluye: "las políticas, directrices y regulaciones sobre prevención de amenazas y riesgos naturales, el señalamiento y localización de las áreas de riesgo para asentamientos humanos, así como las estrategias de manejo de zonas expuestas a amenazas y riesgos naturales" (Congreso de Colombia, 1997, p.7).

De esta manera, la gestión del riesgo de desastres está incluida dentro de tres componentes que estructura los planes de ordenamiento territorial. El componente general del POT establece los objetivos, estrategias y contenidos estructurales de largo plazo, donde se identifica y localiza las acciones y estrategias que se efectuaran sobre el territorio, así como las políticas de ocupación, aprovechamiento y manejo del suelo y de recursos naturales. El componente urbano y el componente rural destacan las políticas, acciones, programas y normas para el desarrollo físico urbano y rural. En estos se debe incluir la delimitación y expansión urbana y rural, las áreas de conservación y protección de los recursos naturales, paisajísticos, así como de las áreas expuestas a amenazas y riesgos naturales. El componente urbano debe incluir los programas y estrategias donde se realizarán procesos de relocalización de comunidades ubicadas en zonas de riesgo de desastres (Congreso de Colombia, 1997).

Según el Banco Mundial (2012), en Colombia los esfuerzos por avanzar en el ordenamiento territorial han sido muy importantes pero aún no son suficientes, ni han logrado los impactos deseados. El reto de lograr una eficiente y eficaz gestión del riesgo no es fácil por los retos que, ante todo, conllevan poder generar estrategias de ordenamiento territorial. territarias 36

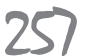


El ordenamiento territorial es fundamental para resolver los conflictos del uso del suelo, que han transformado las condiciones naturales del territorio, afectando principalmente la biodiversidad, la cual se ha visto disminuida por el inadecuado uso y ocupación del suelo, generando graves impactos ambientales como la alteración de la dinámica de las cuencas hidrográficas. A esto se suma la pérdida de cobertura vegetal producto de la deforestación, que junto con el cambio de la dinámica hidrológica y meteorológica han conllevado al aumento de riesgo de desastres naturales. Así mismo, el ordenamiento territorial debe buscar soluciones al crecimiento demográfico, la concentración urbana y al aumento de los niveles de exposición de la población, así como los niveles de vulnerabilidad social generados principalmente por la pobreza.

Las políticas públicas que pretenden resolver los problemas de pobreza, conservación de la biodiversidad, uso del suelo y desarrollo sostenible se implementan mediante instrumentos de planificación. Según Ramírez \& Rubiano (2009) en Colombia, los principales instrumentos de planificación del territorio son los planes de ordenamiento de cuentas hidrográficas (PONCA) y los planes de ordenamiento territorial (POT), los cuales en su componente regulatorio permiten definir las restricciones y los condicionamientos para un manejo adecuado del riesgo y, en su componente programático, ofrecen una gran oportunidad para incorporar un conjunto de proyectos encaminados a consolidar objetivos estratégicos relacionados con la localización y construcción de edificaciones, así como actividades seguras dentro del territorio.

Adicionalmente, los Planes de Desarrollo son instrumentos ligados al voto programático de las autoridades territoriales mediante los cuales se definen prioridades de inversión y, en consecuencia, tienen una mayor probabilidad de ejecución, por lo que deben contar con indicadores y metas cuantificables relacionadas con el conocimiento, la reducción del riesgo y el manejo de desastres a fin de hacer seguimiento, control y evaluación en sus avances (DNP y ESAP, 2007 citado en Banco Mundial, 2012). Junto a estos instrumentos de planificación existen los Bancos de Proyectos por medio de los cuales los entes territoriales registran los proyectos esenciales para llevar acabo los objetivos plasmados en los instrumentos de planificación territorial. A nivel local, los municipios deben incorporar los componentes de la gestión del riesgo de desastres, plasmados en Plan de Gestión del Riesgo Municipal, dentro del Plan de Ordenamiento Territorial, el Plan de Desarrollo Municipal y el Presupuesto Municipal.

\section{La asociación territorial en la gestión del riesgo, el caso del Área Metropolitana del Valle de Aburrá (AMVA)}

A mediados del siglo XX, Colombia sufre una transformación en las principales ciudades del país enfrentando un crecimiento demográfico que conlleva a una reestructuración del espacio, así como de 
la administración y gestión pública de las ciudades. Este cambio estuvo acompañado de un proceso de industrialización que modificó la matriz de desarrollo económico y la configuración espacial de las regiones y ciudades. El crecimiento de la ciudad y el desarrollo industrial creó nuevos usos de la tierra, expandiendo las periferias donde empezaron a originarse nuevos asentamientos humanos (AMVA, 2007; Aristizábal, Vargas \& Mesa, 2008; Coupé, 2011; Toro, Aristizábal, Mejía, Bedoya \& Hermelin, 2010).

Sobre todo barrios populares localizados en sitios entonces periféricos o en áreas centrales degradadas, lo que contribuye a la consolidación de asentamientos con rasgos comunes: la autoproducción del espacio y de la vivienda, la irregularidad en la tenencia, la precariedad de la construcción y las formas organizativas ligadas a culturas tradicionales (Coupé, 2011, p. 20).

De este modo, el crecimiento de las ciudades y el desarrollo industrial conlleva a una ampliación de la infraestructura de interconexión permitiendo que los límites y periferias se entrecrucen efectuando un fenómeno de conurbación:

Esta situación se observa en la región metropolitana del Valle de Aburrá donde los municipios que limitan con Medellín, Bello y Copacabana al Norte, Envigado e Itagüí al Sur, evidencien la tendencia a conformar una sola urbe y desarrollan estrechas relaciones (Coupé, 2011, p. 21).
Con base en esta transformación territorial, y debido a la generación de nuevos vínculos de desarrollo, crecimiento económico y conurbación, se crea el Área Metropolitana del Valle de Aburrá mediante Ordenanza 34 de 1980. En el AMVA se destaca el papel protagonista del municipio de Medellín, el cual asumió un papel de liderazgo en el proceso de desarrollo e integración regional, siendo fundamental en avances significativos para aumentar la capacidad de acción colectiva en el área metropolitana (Restrepo, 2006). Con una estructura institucional diversificada en el nivel metropolitano y regional, se destaca la Red de Cooperación Internacional de Antioquia y la Agencia de Cooperación e Inversión de Medellín en las cuales, la cooperación entre los municipios fronterizos, el gobierno departamental de Antioquia, los socios de la iniciativa privada y de la sociedad civil organizada, se articulan en una cooperación regional donde se desarrollan varios temas y políticas públicas sectoriales (ACI, 2011; Frey, 2012), inclusive en el área de la gestión del riesgos.

La dinámica acelerada y descoordinada de crecimiento de las ciudades que componen el AMVA, generaron un riesgo alto de desastres que fue evidente frente a las repetidas amenazas ambientales que enfrentó el área metropolitana (Gobernación de Antioquia, 2013). Así mismo, las condiciones de vulnerabilidad principalmente frente a movimientos en masa, inundaciones y sismos están estrechamente ligadas con las condiciones de pobreza de la población, las cuales se pueden identi- territarias 36

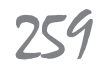


ficar con las necesidades básicas insatisfechas (NBI) (Aristizábal et al., 2008) El porcentaje consolidado de población en condiciones de miseria es de 2,23\% y de pobreza 10,95 (Gobernación de Antioquia, 2013).

Dadas las condiciones de vulnerabilidad del AMVA, la gestión del riesgo de desastres ha sido un eje fundamental de la planificación territorial, que generó un Sistema Metropolitano para la Atención y Recuperación de Desastres del Valle de Aburrá. Este sistema se basa en la implementación de una estrategia conjunta entre los municipios del área metropolitana, por medio de la creación de la Red Metropolitana de Gestión del Riesgo de Desastres - Red Riesgos-. Como instrumentos de la Red, se formuló el Plan Regional de Gestión del Riesgo del Valle de Aburrá y se diseñó un Sistema de Alerta Temprana (SIATA) (Coupé, 2011).

Red Riesgos es un Plan Metropolitano que pretende articular a diferentes actores y entidades tanto públicos como privados y de la sociedad civil en los procesos de conocimiento del riesgo, prevención del riesgo y manejo del desastre. Hace parte fundamental de Red Riesgo el Consejo Municipal de Gestión del Riesgo, conformado por las oficinas de gestión del riesgo de los municipios del área metropolitana, encargado de la coordinación, asesoría, planeación y seguimiento, destinado a garantizar la efectividad y articulación de los procesos de conocimiento del riesgo, reducción del riesgo y manejo del desastre.

\section{tersitarias 36}

El SIATA constituye una de las principales herramientas de conocimiento del riesgo, con las que cuenta las oficinas de gestión del riesgo (DAGRED) de los diferentes municipios del área metropolitana. El objetivo principal del SIATA es alertar de manera oportuna a la comunidad sobre la probabilidad de ocurrencia de un evento hidrometeorológico extremo que pueda generar una situación de emergencia y así reducir los impactos de los fenómenos mediante la implementación de medidas de respuesta ante una amenaza inminente.

Adicionalmente, se destaca la participación ciudadana en Red Riesgos, por medio de organizaciones sociales y comunitarias, las cuales están conformadas por células presentes en los municipios del área metropolitana que forman "La Red Comunitaria de Comités Ambientales", entre los cuales se encuentran los Comités de Prevención y Atención de Desastres Especializado (COPADES), los Comités Educativos de Prevención y Atención de Desastres (CEPAD) y los Comités Ambientales (CUIDA).

La conformación de la Red Comunitaria de Comités Ambientales tiene como objetivo la preparación y participación comunitaria para la prevención y atención de emergencias y desastres, articulando las acciones, estrategias y competencias de sus integrantes y las de aquellas personas que representan a las autoridades ambientales, administraciones municipales y organismos de socorro como Defensa Civil, Cruz Roja y Policía Nacional, que son pilares de apoyo dentro de una estructura integral para 
un grupo especializado en la problemática ambiental y de gestión del riesgo. La Red se caracteriza por los vínculos sociales entre los miembros, donde se comparten intereses, sentido de reciprocidad, sinergia, mecanismos de gestión, articulación, intercambio de información y compromiso con el territorio (Aristizábal et al., 2008).

La complementariedad que tiene el AMVA articulándose con la sociedad en una gestión pública compartida, ha permitido la proliferación de redes como una institución de auto-organización y regulación. Esto mejora la habilidad del Estado de direccionar sus acciones por medio de la ley, la administración de reglas o la regulación de incentivos, por otra parte, proporciona a los ciudadanos y a la sociedad civil la posibilidad de expresar sus temores, deseos y demandas, para poder influir en decisiones políticas sobre el enfrentamiento de los riesgos e implementar interacciones de manera cooperativa en estructuras reticulares de organización y colaboración.

De esta forma, Red Riesgos muestra un rescalonamiento de la estructura del Estado y una articulación entre las escalas del gobierno, redefiniendo las relaciones de trabajo entre el Estado y la sociedad civil. La gestión del riesgo en el AMVA se sustenta en la coordinación de un gobierno en múltiples niveles y escalas, donde el papel del Estado puede considerarse como un agente legitimador de los arreglos institucionales que reducen la vulnerabilidad hacia los desastres naturales.

\section{Conclusiones}

Los principios de la Ley Orgánica de Ordenamiento Territorial sustentan la gestión del riesgo de desastres en Colombia representados principalmente en 1) el empoderamiento de los entes territoriales, sobretodo del nivel municipal, en la gestión del riesgo; 2) la coordinación entre el nivel nacional, departamental y municipal mediante la asignación clara de competencias bajo el Sistema Nacional de Gestión del Riesgo de Desastres; 3 ) el fortalecimiento de Regiones de Planeación y Gestión con el fin de general concurrencia y subsidiaridad entre entes territoriales en los procesos de conocimiento del riesgo, prevención del riesgo y manejo del desastre; 4) el fortalecimiento del departamento como soporte técnico y financiero a los municipios para la complementariedad en los planes de gestión del riesgo; 5) el fortalecimiento del municipio para la Estrategia Municipal para la Respuesta a Emergencias; 6) una visión global y ampliada de los sistemas de gestión del riesgo donde se incluyen tratados y perspectivas internacionales; 7) la modernización de la administración municipal en cuanto a los sistemas de alerta temprana, permitiendo la participación de la sociedad civil. Por tanto, se puede concluir que la Ley Orgánica de Ordenamiento Territorial soporta la institucionalidad del Sistema Nacional de Gestión del Riesgo permitiendo un respaldo financiero a los entes territoriales, promoviendo una asignación de competencias y fortaleciendo territarias 36 261 
la implementación de planes de acción sectorial y territorial.

En Colombia los esfuerzos por avanzar en el ordenamiento territorial han sido muy importantes. El reto de lograr una gestión del riesgo no es fácil porque, ante todo, esto requiere estrategias de ordenamiento territorial. El ordenamiento territorial es fundamental para resolver los conflictos medio ambientales relacionados a las dinámicas de desarrollo y crecimiento económico. Por esto, el ordenamiento territorial debe buscar soluciones al crecimiento demográfico, la concentración urbana y al aumento de los niveles de exposición de la población, así como los niveles de vulnerabilidad social generados principalmente por la pobreza.

\section{Referencias}

Agencia de Cooperación e Inversión de Medellín y el Área Metropolitana (ACI). (2011). Medellín, la región y el mundo: una mirada de la Agencia de Cooperación e Inversión de Medellín y el Área Metropolitana. Medellín: ACI.

AMVA (Área Metropolitana del Valle de Aburrá) INGETEC - IGR. (2007). Diseño del sistema metropolitano para la prevención, atención y recuperación de desastres del Valle de Aburrá. Serie: Conocimientos Metropolitanos Red para la Gestión del Riesgo en el Valle de Aburrá Medellín, Colombia.

territarias 36 262
Valle de Aburrá: Red Riesgos. Revista Gestión y Ambiente, 11(2), 107-122.

Banco Mundial. (2012). Análisis de la gestión del riesgo de desastres en Colombia: un aporte para la construcción de politicas públicas. Bogotá: Banco Mundial.

Becker, A., Chica, C. \& Cárdenas, M. (2003). Ordenamiento Territorial. Reivindicación de la descentralización para el desarrollo. Bogotá: GTZ - FESCOL.

Bonet, J., Pérez, G. \& Ayala, J. (2014). Contexto histórico y evolución del Sistema General de Participaciones en Colombia. Documentos de trabajo sobre economía regional, No 205. Banco de la República, Centro de Estudios Económicos Regionales (CEER) Cartagena, Colombia.

Cabrera, C. \& Naranjo, R. (2003). Las leyes de saneamiento fiscal y su efecto sobre la descentralización. En A. Becker, C. Chica \& M. Cárdenas (Coords.), Ordenamiento Territorial. Reivindicación de la descentralización para el desarrollo (pp. 75-98). Bogotá: GTZ FESCOL.

Congreso de la República de Colombia. (1991). Constitución Política de Colombia.

Colombia, Congreso de la República. Ley 388, por la cual se modifica la Ley 9 de 1989, y la Ley 2 de 1991 y se dictan otras disposiciones con relación al ordenamiento territorial municipal y distrital (18 julio 1997).

Colombia,Congreso de la República. Ley 1454, por la cual se dictan normas orgánicas sobre: ordenamiento territorial

Daniel Calderón Ramírez, Klaus Frey 
y se modifican otras disposiciones (28 junio 2011).

Colombia, Congreso de la República. Ley 1505 , por medio de la cual se crea el sub-sistema nacional de voluntarios de primera respuesta y se otorgan estimulos a los voluntarios de la Defensa Civil, de los cuerpos de Bomberos de Colombia y de la Cruz Roja Colombiana y se dictan otras disposiciones en materia de voluntariado en primera respuesta ( 5 enero 2012).

Colombia, Congreso de la República. Ley 1575 , por medio de la cual se establece la ley general de bomberos de Colombia (21 agosto 2012).

Colombia, Congreso de la República. Ley 1523, por el cual se adopta la Política Nacional de Gestión del Riesgo de Desastres y se establece el Sistema Nacional de Gestión del Riesgo de Desastres y se dictan otras disposiciones (24 abril 2012).

Coupé, F. (agosto, 2011). La gestión del riesgo en el Valle de Aburra, una larga historia. Revista Gestión y Ambiente, 14(2), 17- 44.

Falleti, T.G. (2010). Decentralization and Subnational Politics in Latin America. Cambridge: Cambridge University Press.

Frey, K. (junio, 2012). Abordagens de governança em áreas metropolitanas da América Latina: avanços e entraves. Urbe. Revista Brasileira de Gestão urbana, 4(1), 87-102.

Gobernación de Antioquia. (2013). Anuario Estadístico de Antioquia, 2012 / De- partamento Administrativo de Planeación. Censo General 2005, actualizado a Junio $30 \mathrm{del} 2012$.

Klinke, A. \& Renn, O. (mayo, 2013). A Framework of Adaptive Risk Governance for Urban Planning. Sustainability, 5, 2036-2059, doi:10.3390/su5052036

Manrique, A. (2010). Fundamentos de la organización y del funcionamiento del Estado colombiano (2da Ed.) Bogotá: Editorial Universidad del Rosario.

Ramírez, F. \& Rubiano, D. (2009). Incorporando la gestión del riesgo de desastres en la planificación y gestión territorial. Guía técnica para la interpretación y aplicación del análisis de amenazas y riesgos. Lima: Secretaría General de la CAN - PREDECAN.

Restrepo, C. I. (2006). Medellín una ciudad que se transforma. En J. M. P. Esteve, \& A. F. Paricio (Eds.), La gobernanza democrática: un nuevo enfoque para los grandes retos urbanos y regionales (pp. 92-105). Andalucía: Consejería de Gobernación, Dirección General de Administración Local.

Revéiz, E. (2013). El Estado estratega para el Ordenamiento Territorial. Bogotá: Academia Colombiana de Ciencias Económicas.

Toro, M., Aristizábal, E., Mejía, L., Bedoya, G. \& Hermelin, M. (2010). Red comunitaria para la gestión ambiental en el Valle de Aburrá. Revista Gestión y Ambiente, 13(1), 67-76.

Trujillo, A. (2007). Democracia y territorio: el ordenamiento territorial entre el derecho y la política. Bogotá: Siglo del Hombre territarios 36 263 
Editores y Academia Colombiana de Jurisprudencia.

Unidad Nacional para la Gestión del Riesgo de Desastres. (2012). Formulación del plan municipal de gestión del riesgo. Bogotá: UNGRD.
Unidad Nacional para la Gestión del Riesgo de Desastres. (2013). Guía metodológica para la elaboración de planes departamentales de gestión del riesgo de desastres. Bogotá: UNGRD.

Uribe, E. (1998). Región: punto de fuga. Bogotá: Editorial Universidad de los Andes. 